\title{
MONITORING OF MICROSCOPIC FILAMENTOUS FUNGI IN INDOOR AIR OF TRANSPLANT UNIT
}

\author{
Ondřej Holý1, Ivanka Matoušková1, Alena Kubátová2, Petr Hamal ${ }^{3}$, Lucie Svobodováa ${ }^{3}$ Eva Jurásková4, Luděk \\ Raida $^{5}$ \\ 'Department of Preventive Medicine, Faculty of Medicine and Dentistry, Palacký University Olomouc, Olomouc, Czech Republic \\ ${ }^{2}$ Department of Botany, Faculty of Science, Charles University, Prague, Czech Republic \\ ${ }^{3}$ Department of Microbiology, Faculty of Medicine, Palacký University Olomouc, Olomouc, Czech Republic \\ ${ }^{4}$ Department of Dentistry and Oral Sciences, Faculty of Medicine and Dentistry, Palacký University Olomouc, Olomouc, Czech Republic \\ ${ }_{5}^{5}$ Department of Haemato-Oncology, University Hospital Olomouc, Olomouc, Czech Republic
}

\section{SUMMARY}

Aim: The aim of the study was to control the microbial contamination of indoor air monitored monthly at the Transplant Unit of the University Hospital Olomouc from August 2010 to July 2011.

Methods: The unit is equipped with a three-stage air filtration system with HEPA filters. The MAS-100 air sampler (Merck, GER) was used. Twenty locations were singled out for the purposes of collecting a total of 720 samplings of the indoor air. Swabs of the HVAC diffusers at the sampling locations were always carried out after the sampling of the indoor air.

Results: In total, 480 samples of the indoor air were taken for Sabouraud chloramphenicol agar. In 11 cases $(2.29 \%)$ the cultivation verified the presence of microscopic filamentous fungi. Only two cases involved the sanitary facilities of a patient isolation box; the other positive findings were from the facilities. The most frequent established genus was Aspergillus spp. (4x), followed by Trichoderma spp. (2x) and Penicillium spp. (2x), Paecilomyces spp., Eurotium spp., and Chrysonilia spp. (1x each). In 2 cases the cultivation established sterile aerial mycelium, unfortunately no further identification was possible. A total of 726 swabs of HVAC diffusers were collected ( 2 positive $-0.28 \%$ ). The study results demonstrated the efficacy of the HVAC equipment.

Conclusions: With the continuing increase in the number of severely immunocompromised patients, hospitals are faced with the growing problem of invasive aspergillosis and other opportunistic infections. Preventive monitoring of microbial air contaminants is of major importance for the control of invasive aspergillosis.

Key words: cleanrooms, haemato-oncologic patients, hospital environment, risk of fungal infections

Address for correspondence: 0 . Holý, Department of Preventive Medicine, Faculty of Medicine and Dentistry, Palacký University Olomouc, Hněvotínská 3, 77515 Olomouc, Czech Republic. E-mail: holy.ondrej@seznam.cz.

\section{INTRODUCTION}

The bone marrow or stem cell transplant is a life saving treatment for many cancers and haematological diseases. In 2009, over 2,000 allogeneic and 30,000 autologous stem cell transplantations were carried out worldwide (1). Prior to the transplant, the recipient is granulocytopenic. Peripheral neutrophil numbers are minimal. The patients are hospitalized during the preparatory and the early post-transplant phase. Generally, the engraftment takes 2 to 4 weeks; the duration depends on many factors. The patient (recipient) is placed in an environment with the minimal occurrence of pathogenic and conditionally pathogenic microorganisms. These are special intensive care units with reverse isolation, equipped with three-stage air filtration and positive pressure. The end diffusers designed for the supply of conditioned air are equipped with HEPA filters. The patient stays here at the time of the highest risk of infection, which consists of prolonged neutropenia or damage of mucous membranes, which represent an important barrier $(2,3)$. One way how to reduce the risk of the development of invasive fungal hospital-acquired infections is the monitoring of the microbial contamination of the environment (air, surfaces, water) and microbial colonization of the medical staff, along with effective remedial procedures. The aim of the study was to verify the operation of a three-stage air filtration system and the indoor air quality of the Transplant Unit, Department of Haemato-Oncology, University Hospital Olomouc.

\section{MATERIALS AND METHODS}

The Transplant Unit is designed as a reverse isolation unit with 4 isolation boxes with positive pressure, intended for patients recovering from autologous, allogeneic kin or non-kin transplantation of peripheral stem cells or bone marrow. The unit is air-conditioned with HYD HKBCA 0150 of Nickel Praha (CZE) which works exclusively with fresh air. The air at the unit is treated with a two-stage filtration system with filters Class B and C (EU4 and EU7), heating, cooling and steam humidification. The third stage filtration (filter Class $\mathrm{V}=\mathrm{EU} 12$ ) involves filter laminar ceilings in the rooms. After filtering, heating (cooling) and 
steam-humidifying, the air is driven through the pipeline via filter laminar ceilings ROX LC 24/12 above the patient's bed. From the isolation boxes it is sucked through 4 controlled exhaust TROX DQ diffusers. The sanitary facilities of patient isolation boxes are drained by a radial exhaust fan. Air humidity is regulated with sanitary electric steam generator CONDAIR MATIC MC 560, which is connected to drinking water tap. The cleanliness class of the patient isolation boxes corresponds to that of a super aseptic operating theatre, FED-STD-209E (USA) - 100000 (4). Requirements for the isolation box microclimate are given by values: tiz = $24^{\circ} \mathrm{C}$, til $=21^{\circ} \mathrm{C}$, relative humidity ranging from $50 \%$ to $65 \%$, air replaced 30x per hour, positive pressure against the surrounding area, and volume $34 \mathrm{~m}^{3}$ per patient isolation box. The sanitary facilities of isolation boxes have an exhaust diffuser; air exchange is ensured with a $150 \mathrm{~m}^{3} / \mathrm{h}$, negative pressure against the patient isolation box. The air conditioning system of the Transplant Unit facilities works exclusively with fresh air. Depending on the unit operation needs, the incoming air is heated (cooled), humidified and conditioned with a two-stage filtration system, filters Class $\mathrm{B}$ and $\mathrm{C}$ (EU4 and EU7). The third filtration stage (EU12) is installed in the end of distribution elements - clean hoods F 650 . The air flow rate was based on the same conditions as in patient isolation boxes.

The microbial contamination of the indoor air was monitored monthly at the Transplant Unit, Department of HaematoOncology, University Hospital Olomouc from August 2010 to July 2011. Twenty sampling locations were selected i.e., the patient isolation box, the relevant sanitary facilities and personnel filter in front of the isolation box, 2 sites between the nurse break room and isolation boxes - the hallway, nurse break room, doctor break room, material room, cleaning room, kitchen and the input filter. In the patient isolation box, the air sampler was placed on the dining table, while in the sanitary facility and the personnel filter it was stationed on the floor. At the other sampling locations, it was placed on an auxiliary trolley about 110 $\mathrm{cm}$ above ground. The MAS-100 air sampler (Merck, GER) was used to actively suck 100 litres of the indoor air for 60 seconds. The samplings of the indoor air were always carried out between 8 a.m. and 10 a.m., while the Transplant Unit was in operation. Columbia blood agar (BA) was employed to determine the total number of microorganisms, while Sabouraud glucose agar with chloramphenicol (50 mg/l) (Trios, CZE) was used to identify microscopic filamentous fungi and yeast. A total of 720 samplings of the indoor air were taken. Measurements using the Testo 625 device (Testo AG, GER) focused on the temperature and relative humidity of the indoor air. During the year-long study, the ground floor of the building underwent renovation in March 2011. The incubation was carried out at the temperature of $37^{\circ} \mathrm{C}$ for 48 hours (BA). The final evaluation of the colony growth in Sabouraud glucose agar with chloramphenicol (GPA) took place on the fifth day. The numbers of cultivated colonies were converted with the nomogram and listed as indoor air $\mathrm{CFU} / \mathrm{m}^{3}$ (CFU = colony forming unit). Swabs of the HVAC diffusers at the Transplant Unit rooms were always carried out after the sampling of the microbial contamination of the indoor air. In rectangular diffusers with blades, the samples were collected from blades (3 to 4 items). The individual blades including the circuit of the element were swabbed in swirl diffusers. Smear from the exhaust valve was taken in the area between the solid and the adjustable part, which regulates the amount of supply (exhaust) air. HEPA filters were placed above the patient's bed as ceiling panels. Smear was taken in the area between the frame and the HEPA filter. Smears were carried out using a sterile synthetic swab applicator (COPAN, ITA), soaked in a sterile physiological solution prior to use. Three smears were taken at each diffuser. Then the smears were cultivated in duplicate in Sabouraud broth - TGPA $(5 \mathrm{ml})$, and simultaneously in thioglycollate broth - Thi $(5 \mathrm{ml})$, (Trios, CZE). The incubation was carried out at the temperature of $37^{\circ} \mathrm{C}$ for 5 days (TGPA) and 2 days (Thi), followed by inoculation on solid media (BA/GPA) and their culture. Again, a qualitative method was employed to evaluate the microbial contamination. Cultured colonies of microscopic filamentous fungi were submitted to the Department of Microbiology, Faculty of Medicine and Dentistry, Palacký University Olomouc, for macroscopic and microscopic evaluation. Individual colonies of microscopic filamentous fungi were inoculated onto Sabouraud glucose agar (Trios, CZE), and microcultures were made. Preliminary species identification was performed according to morphology based on direct microscopy. Sections were examined with a light microscope, dry system, with 200x-400x magnification; the shape of the aerial mycelium was evaluated. Further identification was carried out in the Culture Collection of Fungi (Department of Botany, Faculty of Science, Charles University, Prague). The identification was carried out solely on the basis of phenotypic traits, i.e. micro- and macromorphological traits. The sections were cultured on agar media CYA (Czapek Yeast Extract Agar) and MEA (2\% Malt Extract Agar) at $25^{\circ} \mathrm{C}$ in the dark for 10 days (Penicillium chrysogenum Thom, syn. P. notatum) and 1 month (probably Ascomycota spp.). Microsections were observed in lactophenol cotton blue at 400x and 1,000x magnification.

\section{RESULTS}

A total of 240 samplings of the indoor air were collected on Columbia blood agar and 480 samplings of the indoor air on Sabouraud glucose agar with chloramphenicol. In 13 cases the culture proved the presence of microscopic filamentous fungi. Two strains could not be examined any further (grow of sterile mycelium only), while the remaining 11 strains (2.29\%) were identified. Only in two cases microscopic filamentous fungi were identified at the sanitary facilities of a patient isolation box; the other positive findings came from the facilities of the Transplant Unit. The most frequent established strain was Aspergillus spp. (4 cases), followed by 2 cases of Trichoderma spp. and Penicillium spp., while the strains of Paecilomyces spp., Eurotium spp. and Chrysonilia spp. were identified once. Table 1 summarizes the isolates with their sampling location and quantitative evaluation $\left(\mathrm{CFU} / \mathrm{m}^{3}\right.$ of indoor air). All positive culture findings were very low $\left(10 \mathrm{CFU} / \mathrm{m}^{3}\right)$. Total of 726 swabs of HVAC diffusers (inlets, exhausts) were carried out during the year-long monitoring of the microbial contamination of the indoor air. The positive growth of microscopic filamentous fungi in Sabouraud broth was presented in two cases $(0.28 \%)$ - Aspergillus terreus and Chrysonilia sitophila. Both cases were identified in the HVAC diffusers in the hallway. The microbial contamination of surfaces and the staff microbial infestation (smear of the right hand and scalp and swab of both nostrils) were monitored. There was no evidence of 
Table 1. Microscopic filamentous fungi cultured in the indoor air of the Transplant Unit

\begin{tabular}{|c|c|c|c|}
\hline Strain No. & Sampling location & Species & CFU*/m $^{3}$ \\
\hline 1 & physician's office & Trichoderma harzianum AK91/10 & 10 \\
\hline 2 & physician's office & Trichoderma citrinoviride & 10 \\
\hline 3 & hallway near physician's office & Aspergillus terreus & 10 \\
\hline 4 & hallway - rear section & Penicillium chrysogenum & 10 \\
\hline 5 & input filter & Aspergillus sydowii & 10 \\
\hline 6 & input filter & Aspergillus versicolor & 10 \\
\hline 7 & input filter & Aspergillus sydowii & 10 \\
\hline 8 & room 4 - sanitary facilities & Paecilomyces spp. & 10 \\
\hline 9 & room 4 - sanitary facilities & not specified & 10 \\
\hline 10 & room 2 - sanitary facilities & Eurotium amstelodami & 10 \\
\hline 11 & hallway - rear section & Chrysonilia sitophila & 10 \\
\hline 12 & room 4 - sanitary facilites & not specified & \\
\hline 13 & physician's office & Penicillium chrysogenum & \\
\hline
\end{tabular}

${ }^{*} \mathrm{CFU}$ - colony forming units

Table 2. Measured temperature and relative humidity of the indoor air

\begin{tabular}{|l|c|c|}
\hline & Background facilities & Isolation boxes $^{\text {a }}$ \\
\hline Indoor air temperature $\left({ }^{\circ} \mathrm{C}\right)$ & $24.2 \pm 0.9(21.8-26.3)$ & $26.3 \pm 0.7(21.8-25.8)$ \\
\hline Relative humidity of the indoor air $(\%)$ & $40.8 \pm 14.4(16.6-68.1)$ & $40.0 \pm 11.1(19.3-57.4)$ \\
\hline
\end{tabular}

${ }^{a}$ Isolation box includes pateint room and sanitary facility.

microscopic filamentous fungi or yeast from swab samples. The culture results of the indoor air quality at the Transplant Unit, held in March, demonstrated no changes that would indicate an increased presence of the spores of Aspergillus spp. or other microscopic filamentous fungi. Table 2 shows the arithmetic means and standard deviation of the measured temperature and relative humidity of the indoor air. Figures in brackets indicate the range of the measured values. For the purposes of this data, the whole Transplant Unit was divided into the patient isolation box including its sanitary facilities and the personnel filter in front of the isolation box. All remaining rooms are grouped as "background facilities".

\section{DISCUSSION}

The invasive fungal diseases (IFD) are an important cause of morbidity and mortality in immunocompromised patients, especially patients recovering from allogeneic haematopoietic stem cell transplantation (HSCT). IFD prophylaxis includes two basic procedures: regime measures at departments and pharmacological prophylaxis (5). IFD in haemato-oncological patients can be prevented at the time of the highest risk (prolonged neutropenia, immunosuppressive therapy) with a special accommodation regime. This regime contains reverse isolation boxes with positive pressure - cleanrooms - with effective HVAC equipment and built-in HEPA filters (6). Some studies refer to protected environment $(\mathrm{PE})$ which is primarily designed for patients recovering from allogeneic HSCT (7). The legislation or technical standards give technical parameters of isolation boxes with positive (negative) pressure, which are equipped with HEPA filters (8). When the ground floor of the building was being renovated (March 2011), no increased presence of microscopic filamentous fungi spores was detected at the Transplant Unit. Throughout the entire study we found no representatives of microscopic filamentous fungi in the indoor air or on the surfaces of any four isolation boxes. However, the indoor air of the sanitary facilities at the isolation box No. 2 contained Eurotium amstelodami. The most frequent is an exogenous pathogen transfer to the host through air from an inanimate vehicle. As the sanitary facility is only equipped with an indoor air exhaust, it is likely that the strain was introduced into the environment in connection with the operation. The Paecilomyces spp. was found at the sanitary facility of the isolation box No. 4 . The Chrysonilia sitophila strain is found predominantly in plants and only rarely in connection with humans. There have been cases of occupational asthma in individuals who are involved in coffee processing (9). During the study, the strains of Trichoderma harzianum, Trichoderma citrinoviride and Penicillium chrysogenum were cultured in the indoor air of the physician's office. The strains of Trichoderma harzianum and Trichoderma citrinoviride are regarded as an emerging cause of serious fungal infections in immunocompromised especially, haemato-oncological patients (10). The Penicillium spp. has low pathogenicity. Infections caused by Penicillium chrysogenum strain have been established in immunosuppressed patients (11) and rarely in immunocompetent individuals (12). The most commonly isolated microscopic filamentous fungus was Aspergillus spp. Ranking among saprophytes, Aspergillus spp. are ubiquitous in the environment, making up to $40 \%$ of fungal flora in both households and hospitals. During building renovations conidia (asexual spores) are released into the air. When building renovation takes place in health care centres, immunosuppressed patients face the risk of developing invasive pulmonary aspergillosis $(2,13,14)$. In our study we have cultivated three Aspergillus sp. strains. Analysis identified the presence of 
Aspergillus terreus in the hallway between isolation boxes and a nurse break room. While the indoor air at this location was being sampled, new LCD screen wrapped in corrugated paper were stored here. The decontamination of porous materials may not always be perfect. A higher occurrence of $A$. terreus in both the environment and clinical material is pointed out in studies from the USA and selected European countries. Emphasis is placed especially on its in vitro resistance to amphotericin B $(15,16)$. Aspergillus sydowii and Aspergillus versicolor have been established in the input filter. Literature describes cases of onychomycosis or peritonitis in connection with peritoneal dialysis (17-19). These findings could be related to the clogs of all the staff, which are removed here. We consider the three Aspergillus strains detected in the indoor air of the input filter a potential risk for environmental contamination of the Transplant Unit. The input filter is critical due to the fact that all the staff change into sterile personal protective equipment in an area of about $9 \mathrm{~m}^{2}$. The "clean" and "non-clean" sections of this unit come into very close contact. The air is exchanged only through the exhaust and comes in only when the door opens. In addition, this room serves as a passage for an insulated carrier of food for patients and the staff. In order to reduce the risk of microscopic filamentous fungi in these premises, we recommend installing a vertical UV lamp with a cover by the entrance door, which could be in use even in the presence of people. Monitoring of the concentrations of bacteria and microscopic filamentous fungi in the indoor air is useful not only in connection with the incidence of a disease, the symptoms related to staying in the particular environment, but also as preventive screening $(20,21)$. Indoor air sampling of cleanrooms is essential for the air quality monitoring. The previous research shows that microorganisms detected on solid surfaces are not always identical with microorganisms found in the indoor air. Humans can only inhale microorganisms from bioaerosols (22-24).

\section{CONCLUSIONS}

With the continuing increase in the number of severely immunocompromised patients, hospitals are faced with the growing problem of invasive aspergillosis and other opportunistic infections. Because treatment of aspergillosis is difficult and outcome is often fatal, preventive monitoring of microbial air contaminants is of major importance for the control of invasive aspergillosis. Future research should be designed to further elucidate the many questions that remain on the role of the environment in the development of invasive aspergillosis in immunocompromised patients.

\section{Conflict of Interests}

None declared

\section{Acknowledgement}

The project was supported by the Research Support Foundation, Vaduz - grant project no. 801100021/39 - Surveillance of Infectious Complications in Haemato-Oncological Patients.

\section{REFERENCES}

1. Paitoonpong L, Neofytos D, Cosgrove SE, Perl T.M. Infection, Prevention and Control in Hematopoietic Stem Cell - chapter 59. In: Mayhall CG, editors. Hospital epidemiology and infection control. 4th ed. Philadelphia: Wolters Kluwer; 2012. p. 836-72.

2. Lee LD, Hachem RY, Berkheiser M, Hackett B, Jiang Y, Raad II. Hospital environment and invasive aspergillosis in patients with hematologic malignancy. Am J Infect Control. 2012;40(3):247-9.

3. Majhail NS, Rizzo JD, Lee SJ, Aljurf M, Atsuta Y, Bonfim C, et al. Recommended screening and preventive practices for long-term survivors after hematopoietic cell transplantation. Bone Marrow Transplant. 2012;47(3):337-41.

4. Federal Standard 209E: airborne particulate cleanliness classes in cleanrooms and clean Zones [Internet]. Mount Prospect (IL): Institute of Environmental Sciences and Technology; 1992 [cited 2015 May 15]. Available from: https://www.wbdg.org/ccb/FEDMIL/fedstd209e.pdf.

5. Cetkovský P. Profylaxe invazivních mykotických onemocnění u hematologických nemocných. Farmakoterapie. 2011;7(3):365-6.

6. Saravia SA, Raynor PC, Streifel AJ. A performance assessment of airborne infection isolation rooms. Am J Infect Control. 2007;35(5):324-31.

7. Alangaden GJ. Nosocomial fungal infections: epidemiology, infection control, and prevention. Infect Dis Clin North Am. 2011;25(1):201-25.

8. HVAC design manual for hospitals and clinics. Atlanta: American Society of Heating, Refrigerating and Air-Conditioning Engineers; 2003.

9. Francuz B, Year H, Geraut L, Bensefa-Colas L, Nghiem ZH, Choudat D. Occupational asthma induced by Chrysonilia sitophila in a worker exposed to coffee grounds. Clin Vaccine Immunol. 2010;17(10):1645-6.

10. Kviliute R, Paskevicius A, Gulbinovic J, Stulpinas R, Griskevicius L. Nonfatal Trichoderma citrinoviride pneumonia in an acute myeloid leukemia patient. Ann Hematol. 2008;87(6):501-2.

11. D`Antonio D, Violante B, Farina C, Sacco R, Angelucci D, Masciulli $\mathrm{M}$, et al. Necrotizing pneumonia caused by Penicillium chrysogenum. J Clin Microbiol. 1997;35(12):3335-7.

12. Barcus AL, Burdette SD, Herchline TE. Intestinal invasion and disseminated disease associated with Penicillium chrysogenum. Ann Clin Microbiol Antimicrob. 2005;4:21.

13. Pini G, Faggi E, Donato R, Sacco C, Fanci R. Invasive pulmonary aspergillosis in neutropenic patients and the influence of hospital renovation. Mycoses. 2008;51(2):117-22.

14. Bergeron V, Chalfine A, Misset B, Moules V, Laudinet N, Carlet J, et al. Supplemental treatment of air in airborne infection isolation rooms using high-throughput in-room air decontamination units. Am J Infect Control. 2011;39(4):314-20.

15. Rüping MJ, Gerlach S, Fischer G, Lass-Flörl C, Hellmich M, Vehreschild JJ, et al. Environmental and clinical epidemiology of Aspergillus terreus: data from a prospective surveillance study. J Hosp Infect. 2011;78(3):22630.

16. Pemán J, Salavert M. General epidemiology of invasive fungal disease. Enferm Infecc Microbiol Clin. 2012;30(2):90-8. (In Spanish.)

17. Chiu YL, Liaw SJ, Wu VC, Hsueh PR. Peritonitis caused by Aspergillus sydowii in a patient undergoing continuous ambulatory peritoneal dialysis. J Infect. 2005;51(3):e159-61.

18. Takahata Y, Hiruma M, Sugita T, Muto M. A case of onychomycosis due to Aspergillus sydowii diagnosed using DNA sequence analysis. Mycoses. 2008;51(2):170-3.

19. Veraldi S, Chiaratti A, Harak H. Onychomycosis caused by Aspergillus versicolor. Mycoses. 2010;53(4):363-5.

20. Perfect JR, Cox GM, Lee JY, Kauffman CA, de Repentigny L, Chapman $\mathrm{SW}$, et al. The impact of culture isolation of Aspergillus species: a hospitalbased survey of aspergillosis. Clin Infect Dis. 2001;33(11):1824-33.

21. Bénet T, Nicolle MC, Thiebaut A, Piens MA, Nicolini FE, Thomas X, et al. Reduction of invasive aspergillosis incidence among immunocompromised patients after control of environmental exposure. Clin Infect Dis. 2007;45(6):682-6.

22. Menotti J, Waller J, Meunier O, Letscher-Bru V, Herbrecht R, Candolfi E. Epidemiological study of invasive pulmonary aspergillosis in a haematology unit by molecular typing of environmental and patient isolates of Aspergillus fumigatus. J Hosp Infect. 2005 May;60(1):61-8.

23. Alberti C, Bouakline A, Ribaud P, Lacroix C, Rousselot P, Leblanc T, et al. Relationship between environmental fungal contamination and the incidence of invasive aspergillosis inhaematology patients. J Hosp Infect. $2001 \mathrm{Jul} ; 48(3): 198-206$.

24. Faure O, Fricker-Hidalgo H, Lebeau B, Mallaret MR, Ambroise-Thomas $\mathrm{P}$, Grillot R. Eight-year surveillance of environmental fungal contamination in hospital operating rooms and haematological units. J Hosp Infect. 2002 Feb;50(2):155-60. 